\title{
Does the mass of a black hole decrease due to the accretion of phantom energy?
}

\author{
Changjun $\mathrm{Gad}^{*}$ and Xuelei Chen \\ The National Astronomical Observatories, Chinese Academy of Sciences, Beijing, 100012, China \\ Valerio Faraon周 \\ Physics Department, Bishop's University, 2600 College Street, Sherbrooke, Québec, Canada J1M 1Z7 \\ You-Gen Shen§ \\ Shanghai Astronomical Observatory, Chinese Academy of Sciences, Shanghai 200030, China and \\ Joint Institute for Galaxy and Cosmology of SHAO and USTC, Shanghai 200030, China
}

(Dated: November 3, 2018)

\begin{abstract}
According to Babichev et al., the accretion of a phantom test fluid onto a Schwarzschild black hole will induce the mass of the black hole to decrease, however the backreaction was ignored in their calculation. Using new exact solutions describing black holes in a background Friedmann-RobertsonWalker universe, we find that the physical black hole mass may instead increase due to the accretion of phantom energy. If this is the case, and the future universe is dominated by phantom dark energy, the black hole apparent horizon and the cosmic apparent horizon will eventually coincide and, after that, the black hole singularity will become naked in finite comoving time before the Big Rip occurs, violating the Cosmic Censorship Conjecture.
\end{abstract}

\section{INTRODUCTION}

The first year WMAP data combined with the $2 \mathrm{dF}$ galaxy survey and the supernova Ia data favor the phantom energy equation of state of the cosmic fluid $w \equiv$ $P / \rho<-1$ (where $\rho$ and $P$ are the cosmic energy density and pressure, respectively) over the Einstein cosmological constant $(w=-1)$ and the quintessence field $(w>-1)$ [1, 2]. The interpretation of this result brings about the possibility that dark energy could be in the form of a phantom field [3, 4, 5]. Compared to the Lagrangian density of a quintessence field $\mathscr{L}_{\text {quintessence }}=$ $\frac{1}{2} \partial_{\mu} \phi \partial^{\mu} \phi+V(\phi)$, the Lagrangian density of the phantom field has a kinetic energy term with the "wrong" sign, i.e., $\mathscr{L}_{\text {phantom }}=-\frac{1}{2} \partial_{\mu} \phi \partial^{\mu} \phi+V(\phi)$. This "wrong" sign endows the phantom model of dark energy with two surprising properties. First, the density of phantom matter increases with the expansion of the universe in such a way that both the scale factor and the phantom energy density can become infinite in a finite comoving time. If this happens, every object in the universe, including galaxies, stars, the earth and nuclei will be torn apart in a finite future in a so-called Big Rip singularity [3, 6, 7]. A second surprising property of the phantom field was found by Babichev, Dokuchaev and Eroshenko [8], who showed that accretion of phantom energy will induce the mass of black holes to decrease. They concluded that the masses of all black holes will vanish before the Big Rip is reached. However, in their calculations, they ignored the backreaction of phantom matter on the black hole

\footnotetext{
*Electronic address: gaocj@bao.ac.cn

${ }^{\dagger}$ Electronic address: xuelei@cosmology.bao.ac.cn

${ }^{\ddagger}$ Electronic address: vfaraoni@ubishops.ca

$\S$ Electronic address: ygshen@center.shao.ac.cn
}

metric. In a low matter density background, this effect can be safely ignored, but if the matter density of the background is large, for example if it becomes comparable to the black hole density $\sim 1 / M_{0}^{2}\left(M_{0}\right.$ is the black hole mass), the metric describing this black hole will be modified significantly; hence, the backreaction must be accounted for in this case.

The purpose of the present paper is to point out that, taking into account the backreaction, the physical black hole mass may not decrease but will rather increase due to the accretion of phantom matter. The size of the black hole apparent horizon ( $\mathrm{AH}$ ) increases, while that of the cosmic AH decreases, during the evolution of the universe to a Big Rip. There exists a moment $t_{*}$ at which the black hole AH coincides with the cosmological AH, and after which the black hole singularity becomes naked. A naked singularity is forbidden by the Cosmic Censorship Conjecture [9]; in a broad sense, if the latter is correct, it seems that the Cosmic Censor disallows the existence in nature of not only naked singularities, but also of the cryptic phantom field.

In order to study this issue we use new exact solutions of the Einstein field equations describing dynamical black holes embedded in a Friedmann-RobertsonWalker (FRW) universe driven by phantom energy and accreting this cosmic fluid. Independent motivation for the development of dynamical black hole solutions comes from various areas of theoretical physics including black hole thermodynamics, quantum gravity, and mathematical physics [10, 11, 12, 13, 14, 15]. Similar issues about the accretion of phantom energy onto wormholes were discussed in [16].

We use units in which $c=G=\hbar=1$ and this paper is organized as follows: in Sec. II we discuss how the McVittie solution can not describe a black hole embedded in a FRW background universe. Then, we turn to new solutions and show that a new metric found (but not 
studied in detail) in [17] can describe a dynamical black hole in a universe driven by dark matter, radiation, or dark energy. In Sec. III, using the solution of [17], we derive the result that the black hole mass will increase due to the accretion of phantom matter. Secs. IV and VI find the same result for qualitatively different classes of exact solutions, while Sec. VI contains a discussion and the conclusions.

\section{NEW COSMOLOGICAL BLACK HOLE SOLUTIONS}

To investigate the relation between cosmic expansion and local physics, McVittie introduced in 1933 his wellknown solution [18]

$$
\begin{aligned}
d s^{2}= & -\frac{\left[1-\frac{M_{0}}{2 a(t) r}\right]^{2}}{\left[1+\frac{M_{0}}{2 a(t) r}\right]^{2}} d t^{2}+a^{2}(t)\left[1+\frac{M_{0}}{2 a(t) r}\right]^{4} \\
& \cdot\left(d r^{2}+r^{2} d \Omega^{2}\right)
\end{aligned}
$$

where $d \Omega^{2}$ is the line element on the unit 2-sphere and the constant $M_{0}$ denotes the physical mass of the black hole when $a(t)=$ const. (this can be seen by rescaling the radial coordinate as $\tilde{r} \equiv a r)$. The scale factor $a(t)$ is an arbitrary (positive) function of the comoving time $t$. This metric reduces to the familiar spatially flat FRW metric as $M \rightarrow 0$. So at a first glance, the metric seems to represent a Schwarzschild black hole embedded in a spatially flat FRW universe [19]. However it is argued that, in general, the McVittie solution can not describe a black hole because it is singular on the 2-sphere $r=M_{0} / 2$ (which reduces to the Schwarzschild horizon if $a=$ const.) [20] and this singularity is spacelike [21]. It is also claimed that the McVittie metric may describe a point mass located at $r=0$ and embedded in a FRW universe. However, this point mass is, in general, surrounded by the singularity at $r=M_{0} / 2$, which is difficult to interpret. This singularity was studied in Refs. 20]. Nolan [22] showed that this is a weak singularity in the sense that the volume of an object approaching the $r=M_{0} / 2$ surface is not shrunk to zero, and therefore the energy density of the cosmic fluid remains finite. On the other hand, the pressure diverges at $r=M_{0} / 2$ together with the Ricci scalar $R[20,23]$. So, while the McVittie metric represents some kind of strongly gravitating central object, its physical interpretation is not totally clear and remains the subject of debate [20, 21]. The only exception is the Schwarzschild- de Sitter (or Köttler) metric, which corresponds to a special case of the McVittie metric and represents a genuine black hole of constant mass embedded in a de Sitter background [17, 20, 22].

Because the McVittie solution can not describe a black hole in a FRW universe (with the exception of the Schwarzschild-de Sitter one), we turn our attention to the new solutions of Ref. [17], described by the generalization of the McVittie metric 24]

$$
\begin{aligned}
d s^{2}= & -\frac{\left[1-\frac{M(t)}{2 a(t) r}\right]^{2}}{\left[1+\frac{M(t)}{2 a(t) r}\right]^{2}} d t^{2}+a(t)^{2}\left[1+\frac{M(t)}{2 a(t) r}\right]^{4} \\
& \cdot\left(d r^{2}+r^{2} d \Omega^{2}\right)
\end{aligned}
$$

in the background of an imperfect fluid with a radial heat flux and, possibly, a radial mass flow simulating accretion onto a black hole embedded in a generic FRW universe.

In the McVittie metric, the mass coefficient $M$ is constant, which expresses the assumption (hereafter referred to as the "McVittie condition") that $G_{0}^{1}=0$, where $G_{\mu \nu}$ is the Einstein tensor, and therefore to vanishing radial mass flow onto the central object, $T_{0}^{1}=0$, and to no accretion. $M(t)$ corresponds [17] to the Hawking-Hayward quasi-local mass of the metric (Eq. 2) and, therefore, the McVittie no-accretion condition corresponds to constant physical mass of the central object, which is static even when the universe expands or contracts. Here instead, following [17], the black hole is allowed to accrete cosmic fluid and $M(t)$ changes with time.

Two distinct processes concur in the time variation of the black hole mass: the first is the radial flow of energy onto the black hole due to the radial motion of the cosmic fluid toward the horizon (this is a simplified version of the accretion process considered by Babichev et al.), possibly supplemented by a radial heat flux, which necessarily requires an imperfect fluid description. The second process is the variation of the mass enclosed by the black hole apparent horizon due to the motion of the horizon itself; this process is absent in the McVittie solution (in which the singular surface $r=M_{0} / 2$ does not expand) and in the static black hole considered by Babichev et $a l$. , but is present for any dynamical horizon even in the absence of the first accretion process. However, unless the total mass of the central object is zero (which could happen, e.g., for a spherical shell of exotic matter), a radial energy flow is to be expected due to its gravitational pull. The solutions of [17] exhibit accretion onto the central black hole due to radial mass flow and/or to a radial heat flux.

The components of the Einstein tensor for the metric (2) are [17]

$$
\begin{aligned}
& G_{0}^{0}=-\frac{3 A^{2}}{B^{2}}\left(\frac{\dot{a}}{a}+\frac{\dot{m}}{r A}\right)^{2}, \\
& G_{0}^{1}=-\frac{2 m}{r^{2} a^{2} A^{5} B}\left(\frac{\dot{a}}{a}+\frac{\dot{m}}{m}\right), \\
& G_{1}^{1}=G_{2}^{2}=G_{3}^{3}=-\frac{A^{2}}{B^{2}}\left\{2 \frac{d}{d t}\left(\frac{\dot{a}}{a}+\frac{\dot{m}}{r A}\right)\right. \\
& \left.+\left(\frac{\dot{a}}{a}+\frac{\dot{m}}{r A}\right)\left[3\left(\frac{\dot{a}}{a}+\frac{\dot{m}}{r A}\right)+\frac{2 \dot{m}}{r A B}\right]\right\},
\end{aligned}
$$


where $A \equiv 1+\frac{m}{2 r}, B \equiv 1-\frac{m}{2 r}$, and $m(t) \equiv M(t) / a(t)$. Assuming that there is no mass flow but that there is a radial heat flux in the stress-energy tensor

$$
T_{\mu \nu}=(P+\rho) u_{\mu} u_{\nu}+P g_{\mu \nu}+q_{\mu} u_{\nu}+q_{\nu} u_{\mu},
$$

where $u^{\mu}=\left(\left|-g_{00}\right|^{-1 / 2}, 0,0,0\right)$ and $q^{\mu}=(0, q, 0,0)$, the Einstein equations are

$$
\begin{aligned}
& G_{0}^{1}=-8 \pi \frac{A}{B} q, \\
& G_{0}^{0}=-8 \pi \rho, \\
& G_{1}^{1}=G_{2}^{2}=G_{3}^{3}=8 \pi P .
\end{aligned}
$$

For the constant equation of state $P=w \rho$ ( $w=$ const. $)$, combined with the $(0,0)$ and $(1,1)$ Einstein equations to obtain $G_{1}^{1}+w G_{0}^{0}=0$, one derives

$$
\begin{aligned}
& 2 \frac{d}{d t}\left(\frac{\dot{a}}{a}+\frac{\dot{m}}{r A}\right)+\left(\frac{\dot{a}}{a}+\frac{\dot{m}}{r A}\right)\left[3(w+1)\left(\frac{\dot{a}}{a}+\frac{\dot{m}}{r A}\right)\right. \\
& \left.+\frac{2 \dot{m}}{r A B}\right]=0
\end{aligned}
$$

and

$$
q=\frac{-\dot{M}}{4 \pi r^{2} a^{3} A^{6}},
$$

which expresses the fact that an ingoing flow $(q<0)$ corresponds to an increase of the quasi-local mass $\dot{M}>0$, and vice-versa.

As $r \rightarrow+\infty$, the scale factor of the universe is given by the familiar expressions

$$
\begin{aligned}
& a(t)=a_{0}\left(t_{\text {rip }}-t\right)^{\frac{2}{3(w+1)}} \quad \text { if } w<-1, \\
& a(t)=a_{0} \mathrm{e}^{H_{0} t} \quad \text { if } w=-1, \\
& a(t)=a_{0} t^{\frac{2}{3(w+1)}} \quad \text { if } w>-1,
\end{aligned}
$$

where $t_{\text {rip }}$ denotes the Big Rip. It is easy to see that the previous form of $a(t)$ with the choice $M(t)=M_{0} a(t)$ (with $M_{0}$ a positive constant) and the corresponding heat flux density

$$
q=\frac{-M_{0} H}{4 \pi r^{2} a^{2} A^{6}},
$$

constitutes a solution of the full Einstein equations. The energy density and pressure depend on both the radius and the comoving time, $\rho=\rho(t, r), P=P(t, r)$, and

$$
\rho(t, r)=\frac{3 A^{2}}{B^{2}}\left(\frac{\dot{a}}{a}+\frac{\dot{m}}{r A}\right)^{2}
$$

is non-negative.

Thanks to the particular form of the metric coefficient $M(t)$, this solution is conformal to a Schwarzschild black hole with conformal factor equal to the scale factor of the background universe $a(t)$ [15, 17], a feature shared with the Sultana-Dyer solution 25] ( $c f$. also the solutions of Ref. [23]). The mass coefficient $M(t)$ coincides with the Hawking-Hayward quasi-local mass of the spacetime (Eq2), which should be identified with the physical black hole mass [15]. The fact that the latter increases in an expanding universe describes the constant accretion due to the radial energy flux from the surrounding fluid. The McVittie condition $G_{0}^{1}=0$ equivalent to $\dot{M}=0$ that forbids accretion onto the central object in the McVittie metric is clearly not imposed in the solutions of [17] that we use here, allowing accretion to occur.

These solutions describe a cosmological black hole embedded in a FRW background and present advantages over the previous Sultana-Dyer 25] and McClure-Dyer 23 solutions, in the sense that both the energy density and the pressure are finite near the black hole horizon, and the energy density is positive-definite [17].

More general solutions with a different form of the mass coefficient and no conformal Killing horizon are considered later in Sec. IV.

\section{VIOLATION OF COSMIC CENSORSHIP}

The exact solution describing a black hole embedded in a FRW universe that we adopt in this section corresponds to the choice $M(t)=M_{0} a(t)$ and $a(t)$ given by Eq. (12), and it can be written as

$$
\begin{aligned}
d s^{2}= & -\frac{\left(1-\frac{M_{0}}{2 r}\right)^{2}}{\left(1+\frac{M_{0}}{2 r}\right)^{2}} d t^{2}+a^{2}(t)\left(1+\frac{M_{0}}{2 r}\right)^{4} \\
& \cdot\left(d r^{2}+r^{2} d \Omega^{2}\right)
\end{aligned}
$$

in isotropic coordinates. If $a \equiv 1$, then $r=M_{0} / 2$ is the event horizon of the Schwarzschild black hole; however, if $a$ is not constant, $r=M_{0} / 2$ is no longer the event horizon but merely a 2 -sphere enclosed within the $\mathrm{AH}$ and a conformal Killing horizon.

In order to investigate the evolution of the black hole mass and horizon, it is convenient to rewrite Eq. (17) using the areal radius $\tilde{r} \equiv r\left(1+\frac{M_{0}}{2 r}\right)^{2}$. Eq. (17) then becomes

$$
\begin{aligned}
d s^{2}= & -\left(1-\frac{2 M_{0}}{\tilde{r}}\right) d t^{2}+a^{2}\left(1-\frac{2 M_{0}}{\tilde{r}}\right)^{-1} d \tilde{r}^{2} \\
& +a^{2} \tilde{r}^{2} d \Omega^{2}
\end{aligned}
$$

Further setting $R=a \tilde{r}$, Eq. (18) is rewritten in the 
Painlevé-Gullstrand form as

$$
\begin{aligned}
d s^{2}= & -\left[1-\frac{2 M_{0} a}{R}-\left(1-\frac{2 M_{0} a}{R}\right)^{-1} H^{2} R^{2}\right] d t^{2} \\
& +\left(1-\frac{2 M_{0} a}{R}\right)^{-1} d R^{2} \\
& -2 H R\left(1-\frac{2 M_{0} a}{R}\right)^{-1} d t d R+R^{2} d \Omega^{2}
\end{aligned}
$$

The coordinate system $(t, R, \theta, \phi)$ is not orthogonal; the cross-term $d t d R$ can be eliminated by introducing the new time coordinate $\bar{t}$ defined by

$$
d \bar{t}=\frac{1}{F(t, R)}\left[d t+\frac{H R}{\left(1-\frac{2 M_{0} a}{R}\right)^{2}-H^{2} R^{2}} d R\right],
$$

where $F(t(\bar{t}, R), R)$ is an integrating factor which always exists and solves the partial differential equation

$$
\partial_{R}\left(\frac{1}{F}\right)=\partial_{t}\left[\frac{1}{F} \frac{H R}{\left(1-\frac{2 M_{0} a}{R}\right)^{2}-H^{2} R^{2}}\right]
$$

in order to make $d \bar{t}$ an exact differential. The metric (19) is cast in the Nolan gauge form

$$
\begin{aligned}
d s^{2}= & -\left[1-\frac{2 M_{0} a}{R}-\left(1-\frac{2 M_{0} a}{R}\right)^{-1} H^{2} R^{2}\right] F^{2} d \vec{t}^{2} \\
& +\left[1-\frac{2 M_{0} a}{R}-\left(1-\frac{2 M_{0} a}{R}\right)^{-1} H^{2} R^{2}\right]^{-1} d R^{2} \\
& +R^{2} d \Omega^{2}
\end{aligned}
$$

where $H(t)$ and $F(t, R)$ are now functions of $\bar{t}$ and $R$. The Schwarzschild solution in Schwarzschild coordinates is recovered by setting $a \equiv 1$ (and, consequently, $H=0$ and $R=\tilde{r}$ ), while the Schwarzschild-de Sitter solution is recovered by using Eq. (1) and Eq. (13); further, the latter reduces to the de Sitter solution (in static coordinates) if one also sets $M_{0}=0$.

In the Schwarzschild-de Sitter spacetime, there exists a black hole apparent horizon $(\mathrm{AH})$ and a cosmic $\mathrm{AH}$. The metric (22) describes a spherically symmetric and dynamical black hole in a FRW background more general than de Sitter. In this case, the event horizon may not be well-defined, but the $\mathrm{AH}$ always exists. The $\mathrm{AH}$ is a marginally trapped surface with vanishing expansion and has been argued to be a causal horizon for a dynamical spacetime. The $\mathrm{AH}$ is associated with the Hawking temperature, gravitational entropy and other thermodynamical aspects [10, 11, 12, 13]. The first law of thermodynamics for the $\mathrm{AH}$ has been derived not only in general relativity but also in several other theories of gravity including the Lovelock, nonlinear, scalar-tensor, and braneworld theories [26, 27, 28, 29, 30, 31, 32]. In view of this point, in order to investigate the evolution of the black hole mass, we calculate the energy contained inside the $\mathrm{AH}$. To this end, let us first calculate the radius of the black hole AH: Eq. (22) yields

$$
1-\frac{2 M_{0} a}{R} \mp H R=0,
$$

as the equation of the $\mathrm{AH}$ (this expression appears also in Ref. [14], and it can also be derived from Eq. (17) by replacing $r$ with $R$ ). We discard the solution corresponding to the lower sign and to a negative radius, then Eq. (23) has the two physical roots

$$
\begin{aligned}
& R_{c}=\frac{1}{2 H}\left(1+\sqrt{1-8 M_{0} \dot{a}}\right), \\
& R_{b}=\frac{1}{2 H}\left(1-\sqrt{1-8 M_{0} \dot{a}}\right),
\end{aligned}
$$

where an overdot denotes differentiation with respect to the comoving time $t$ and the explicit time-dependence of $H$ and $\dot{a}$ contains the time evolution of the AH in which we are interested. $R_{c}$ and $R_{b}$ identify the cosmic $\mathrm{AH}$ and the black hole $\mathrm{AH}$, respectively. When the radius $R$ crosses the black hole $\mathrm{AH} R_{b}$, the $g_{00}$ and $g_{11}$ terms in the line element (22) change sign, revealing that the $\mathrm{AH}$ is a one-way membrane for particles falling into the black hole. A calculation of the null surface of equation $g^{\mu \nu} \frac{\partial f}{\partial x^{\mu}} \frac{\partial f}{\partial x^{\nu}}=0$ shows that the $\mathrm{AH}$ is a null 2-sphere. The coordinate transformations yield the correspondence

$$
r=M_{0} / 2 \Leftrightarrow \tilde{r}=2 M_{0} \Leftrightarrow R=2 M_{0} a=2 m_{H}(t)(26)
$$

where $m_{H}=M$ is the Hawking-Hayward quasi-local mass (see the end of this section for a discussion). So, the conformal Killing horizon $r=M_{0} / 2$ is no longer the black hole event horizon but is instead a 2-sphere contained inside the AH. In fact, it follows from Eq. (23) (with the upper sign) that the roots of this equation satisfy $R_{c, b}>2 M_{0} a=2 m_{H}$. Moreover, the energy density $\rho$ and the pressure $P$ are both regular on the AH and $\rho$ is positive-definite.

We are interested in the accretion of phantom energy onto the black hole; the black hole $\mathrm{AH}$ and the cosmic AH computed from Eq. (12) are plotted in Fig. (2) for the parameter choice $a_{0}=1, M_{0}=1, t_{\text {rip }}=1$, and $w=-2$. As is clear from this figure, in our solution the black hole $\mathrm{AH}$ increases while the cosmic $\mathrm{AH}$ decreases as the universe evolves. If the future universe is dominated by phantom energy, then there is a time $t_{*}$ at which these horizons coincide, which is given by $\dot{a}\left(t_{*}\right)=\frac{1}{8 M_{0}}$, or

$$
t_{*}=t_{\text {rip }}-\left(\frac{16 a_{0} M_{0}}{3|w+1|}\right)^{\left|\frac{3(w+1)}{3 w+1}\right|} .
$$

At the comoving time $t_{*}$, the critical AH radius is $R_{c r}=$ $\frac{1}{2 H}$. At times $t>t_{*}$ both horizons disappear and the black hole singularity becomes a naked singularity. We stress that this happens before the Big Rip is reached. 
The appropriate notion of mass for a cosmological black hole is the Hawking-Hayward quasi-local mass which, for the metric (2) reduces to $m_{H}(t)=M(t)$ and, for the cosmological black hole (17), to

$$
m_{H}(t)=M_{0} a(t) .
$$

For our solution this is always increasing in an expanding universe, even when the black hole $\mathrm{AH}$ disappears (the computation of this mass does not depend on the presence of an $\mathrm{AH}$ ), a fact that can be interpreted as the energy flow continuing to fall onto the naked singularity after the horizon has disappeared.

The conclusion that the black hole AH and the Hawking mass increase in a phantom-dominated universe running toward the Big Rip is the opposite of what Babichev et al. [8] find by extrapolating rigorous results obtained for the accretion of a phantom test fluid onto a static Schwarzschild black hole. They find that the Schwarzschild mass decreases because the accretion rate $\dot{m} \propto(P+\rho)<0$. However, this result can not be generalized directly to a cosmological, time-dependent, black hole. The reason for the discrepancy is that the HawkingHayward quasi-local mass $m_{H}=M$ (which implies the subtraction of a background energy and reduces to the Schwarzschild mass when $a \equiv 1$ ) depends only on the energy density $\rho$ but not on the pressure $P$ [33]. Since $\rho>0$, it does not matter how negative $P$ is, and $\dot{m}_{H}$ is always positive. The Schwarzschild mass notion is not appropriate, nor defined, for a black hole in an asymptotically FRW spacetime.

The quasi-local mass has been proved to be superior to other notions of mass, which have generated some confusion in the literature, in the study of the Hawking temperature of cosmological black holes 15]. Nevertheless, it may be interesting to try out a different notion of mass. An obvious candidate is the (generalized) Misner-Sharp mass that has been used in black hole thermodynamics in relation with energy flows through the $\mathrm{AH}[14,26]$. While we do not want to fuel a debate on which is the most convenient notion of mass, and for which purposes, we present the time evolution of this generalized Misner-Sharp mass contained inside the AH. For a spherically symmetric space-time with line element $d s^{2}=h_{\mu \nu} d x^{\mu} d x^{\nu}+R^{2} d \Omega^{2}$, the generalized Misner-Sharp mass is $M_{M S}=R\left(1-h^{\mu \nu} R_{, \mu} R_{, \nu}\right) / 2$ [34]. At the AH, it is $h^{\mu \nu} R_{, \mu} R_{, \nu}=0$ and the generalized Misner-Sharp mass inside the AH (also computed in Ref. [14]) is simply

$$
M_{M S}=R_{b} / 2
$$

for all times for which the expression (25) of $R_{b}$ is well-defined. Both the quasi-local and the generalized Misner-Sharp mass of the black hole increase with time: this is at odds with the decreasing mass property found in Ref. [8] (the difference between MisnerSharp and Hawking-Hayward masses can be seen also in the Schwarzschild-de Sitter metric). Eqs. (28) and (29) show that a discussion of the time evolution of black hole

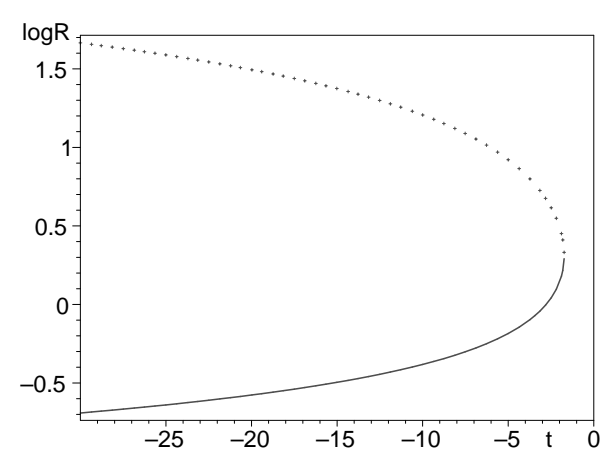

FIG. 1: The black hole apparent horizon (solid line) increases while the cosmic apparent horizon (dotted line) decreases with the accretion of phantom energy onto the black hole. There is a time at which the two horizons coincide and after which both horizons disappear and the black hole singularity becomes a naked singularity. The plots correspond to the equation of state parameter $w=-2$.

masses is meaningless until the correct notion of mass is identified, and the choice is non-trivial for a timedependent cosmological black hole.

\section{A MORE GENERAL CLASS OF SOLUTIONS}

One may wonder whether the conclusions reached in the previous section are due to the peculiar form of the solution employed, in particular the choice of HawkingHayward quasi-local mass scaling as the scale factor, $M(t)=M_{0} a(t)$. The answer is negative, as we shall see below. The exact solution studied in the previous section can be generalized to an arbitrary form of the Hawking mass $m_{H}=M(t)$ [17] (in this case, the 2-sphere $r=M / 2$ is no longer a Killing horizon). Consider again Eq. (2) with a general form of $M(t)$; by defining the areal radius $\tilde{r}=r\left(1+\frac{M(t)}{2 r a(t)}\right)^{2}$ as before and $m(t) \equiv M(t) / a(t)$, the metric (2) is rewritten as

$$
\begin{aligned}
d s^{2}= & -\left[1-\frac{2 m}{\tilde{r}}-\frac{a^{2} \dot{m}^{2}}{1-\frac{2 m}{\tilde{r}}}\left(1+\frac{m}{2 \tilde{r}}\right)^{2}\right] d t^{2} \\
& +a^{2}\left(1-\frac{2 m}{\tilde{r}}\right)^{-1} d \tilde{r}^{2} \\
& +a^{2} \tilde{r}^{2} d \Omega^{2}-\frac{2 \dot{m} a^{2}\left(1+\frac{m}{2 r}\right)}{1-\frac{2 m}{\tilde{r}}} d t d \tilde{r}
\end{aligned}
$$

Introducing the comoving $R \equiv a \tilde{r}$, in terms of which $d r=\frac{d R}{a}-H \tilde{r} d t$, Eq. (30) is turned into the Painlevé- 
Gullstrand form

$$
\begin{aligned}
d s^{2} & =-\left[1-\frac{2 M}{R}-\frac{\left(H R+\dot{m} a \sqrt{\frac{\tilde{r}}{r}}\right)^{2}}{1-\frac{2 M}{R}}\right] d t^{2} \\
& +\frac{d R^{2}}{1-\frac{2 M}{R}}+R^{2} d \Omega^{2} \\
& -\frac{2}{1-\frac{2 M}{R}}\left(H R+\dot{m} a \sqrt{\frac{\tilde{r}}{r}}\right) d t d R .
\end{aligned}
$$

Further setting $A(t, R) \equiv 1-2 M / R$ and $C(t, R) \equiv H R+$ $\dot{m} a \sqrt{\frac{\tilde{r}}{r}}$ and defining the time coordinate $\bar{t}$ by

$$
d \bar{t}=\frac{1}{F}\left(d t+\frac{C}{A^{2}-C^{2}} d R\right),
$$

where $F(\bar{t}, R)$ is again an integrating factor that makes $d \bar{t}$ an exact differential, one has

$$
\begin{aligned}
& d s^{2}=-\frac{A^{2}-C^{2}}{A}\left[F^{2} d \vec{t}^{2}+\frac{C^{2} d R^{2}}{\left(A^{2}-C^{2}\right)^{2}}-\frac{2 F B}{A^{2}-C^{2}} d \bar{t} d R\right] \\
& +\frac{d R^{2}}{A}+R^{2} d \Omega^{2}-\frac{2 C}{A} d R\left(F d \tilde{t}-\frac{C}{A^{2}-C^{2}} d R\right) .
\end{aligned}
$$

The cross terms in $d R d \bar{t}$ cancel out and we are left with the line element in the Nolan gauge

$$
\begin{aligned}
& d s^{2}=-\left(1-\frac{2 M}{R}\right)\left[1-\frac{\left(H R+\dot{m} a \sqrt{\frac{\tilde{r}}{r}}\right)^{2}}{\left(1-\frac{2 M}{R}\right)^{2}}\right] F^{2} d \vec{t}^{2} \\
& +\left(1-\frac{2 M}{R}\right)^{-1}\left[1-\frac{\left(H R+\dot{m} a \sqrt{\frac{\tilde{r}}{r}}\right)^{2}}{\left(1-\frac{2 M}{R}\right)^{2}}\right]^{-1} d R^{2} \\
& +R^{2} d \Omega^{2} .
\end{aligned}
$$

Inspection of this metric locates the horizons at $C= \pm A$, or

$$
H R+\dot{m} a \sqrt{\frac{\tilde{r}}{r}}= \pm\left(1-\frac{2 M}{R}\right) .
$$

Since the Hawking mass is $M(t)=m(t) a(t)$, the expression on the left hand side can be written as

$$
H R+M\left(1+\frac{m}{2 r}\right)\left(\frac{\dot{M}}{M}-H\right)
$$

in which the factor $M\left(1+\frac{m}{2 r}\right)$ measures the deviation of the radius from $2 M$ (with $r>m / 2 \Leftrightarrow R>2 M \Leftrightarrow$ $\left.M\left(1+\frac{2 m}{r}\right)>2 M\right)$, while the factor $\left(\frac{\dot{M}}{M}-H\right)$ is the difference between the percent variation of the black hole mass and that of the scale factor of the cosmic substratum. The vanishing of this factor can be regarded as the analog of the condition for stationary accretion in a time-dependent background (keeping with the spirit of the quasi-local mass definition in which a background quantity is subtracted). Then, the solution considered in the previous section corresponds to stationary accretion relative to the FRW background.

By discarding the lower sign as usual, Eq. (35) becomes

$$
H R^{2}+\left[M\left(1+\frac{m}{2 r}\right)\left(\frac{\dot{M}}{M}-H\right)-1\right] R+2 M=0 .
$$

Due to the general form of the function $M(t)$ (not scaling as $\left.M(t)=M_{0} a(t)\right)$, now the coefficient $\left(1+\frac{m}{2 r}\right)$ appears, in which $r=r(R)$. Due to this dependence, Eq. (37) can not be regarded as a quadratic algebraic equation for the AHs radii as before. Nevertheless, it is instructive to treat it formally as such, obtaining the formal roots

$$
\begin{aligned}
& R_{c, b}=\frac{1}{2 H}\left\{1-M\left(1+\frac{m}{2 r}\right) \frac{\dot{m}}{m}\right. \\
& \left. \pm \sqrt{\left[1-M\left(1+\frac{m}{2 r}\right) \frac{\dot{m}}{m}\right]^{2}-8 m \dot{a}}\right\} .
\end{aligned}
$$

Since $r$ depends on $R$, this is only an implicit equation for the cosmic and black hole $\mathrm{AH}$ radii $R_{c, b}$, but it suffices for our purposes. When the argument of the square root is positive, there are a cosmic $\mathrm{AH}$ at $R_{c}$ and a black hole $\mathrm{AH}$ at $R_{b}$ while, if this argument becomes negative because the time derivative $\dot{a}$ increases without limit as the Big Rip is approached, these AHs coincide at the common critical radius $\sqrt{\frac{2 M}{H}}$, and then disappear leaving behind a naked singularity. This situation occurs, e.g., for the simple choice of the mass function

$$
m(t)=\frac{m_{0}}{a^{\alpha}(t)} \quad(\alpha>1) .
$$

Then, $\left[1-M\left(1+\frac{m}{2 r}\right) \frac{\dot{m}}{m}\right] \rightarrow 1$ as $t \rightarrow t_{\text {rip }}^{-}$and

$$
m \dot{a}=\frac{2 m_{0}}{3|w+1| a_{0}^{\alpha-1}}\left(t_{\text {rip }}-t\right)^{\frac{3 w+2 \alpha+1}{3(w+1)}},
$$

and the heat flux (11) is finite on the AH. If $\alpha<\frac{3|w|-1}{2}$ (which is compatible with both requirements $\alpha>1$ and $w<-1$ ), the horizon disappears but the central mass does not, leaving a naked singularity.

\section{ANOTHER DYNAMICAL PHANTOM BLACK HOLE}

In order to further clarify the issue, in this section we construct another dynamical black hole solution with 
phantom scalar field. To this end, let us first recall the Fonarev solution [35] which is derived from the action

$$
S=\int d^{4} x \sqrt{-g}\left[R-\frac{1}{2} \partial_{\mu} \phi \partial^{\mu} \phi-V(\phi)\right] .
$$

This action describes an Einstein-scalar system. The potential is assumed to be exponential

$$
V(\phi)=V_{0} e^{-\lambda \phi},
$$

where $V_{0}$ and $\lambda$ are two real constants: $\lambda$ describes the slope of the potential and $V_{0}=V(\phi=0)$. Without loss of generality, we assume that they are both positive. This potential has been investigated at length in the context of homogenous cosmology [36]. The Einstein equation and Klein-Gordon equations are

$$
\begin{gathered}
G_{\mu \nu}=\partial_{\mu} \phi \partial_{\nu} \phi-\frac{1}{2} g_{\mu \nu} \partial_{\alpha} \phi \partial^{\alpha} \phi-g_{\mu \nu} V, \\
\nabla_{\alpha} \nabla^{\alpha} \phi-\frac{d V}{d \phi}=0 .
\end{gathered}
$$

The spherically symmetric solution obtained by Fonarev is

$$
\begin{aligned}
d s^{2}= & a(\eta)^{2}\left[-f^{2}(r) d \eta^{2}+\frac{1}{f^{2}(r)} d r^{2}+S(r)^{2} d \Omega^{2}\right] \\
\phi= & \frac{1}{\lambda} \ln \left[\frac{V_{0}\left(\lambda^{2}-2\right)^{2}}{2 a_{0}^{2}\left(6-\lambda^{2}\right)}\right]+\lambda \ln a \\
& +\frac{1}{\sqrt{\lambda^{2}+2}} \ln \left(1-\frac{2 w}{r}\right)
\end{aligned}
$$

where

$$
\begin{aligned}
f & =\left(1-\frac{2 w}{r}\right)^{\frac{\alpha}{2}}, \quad \alpha=\frac{\lambda}{\sqrt{\lambda^{2}+2}}, \\
S^{2} & =r^{2}\left(1-\frac{2 w}{r}\right)^{1-\alpha}, \quad a=a_{0}|\eta|^{\frac{2}{\lambda^{2}-2}} .
\end{aligned}
$$

where $w$ and $a_{0}$ are integration constants. The physical meaning of $w$ will be clear later, while $a_{0}$ is a normalization constant for the scale factor. For simplicity, hereafter we set $a_{0}=1$. When $w=0$, the metric (45) reduces to the FRW universe while, when $a=1, \alpha=1$, it reduces to the Schwarzschild solution. So this solution describes a black hole embedded in FRW universe.

Now, a dynamical phantom black hole solution can be constructed from the Fonarev solution via the transformations

$$
\phi \rightarrow i \phi, \quad \lambda \rightarrow-i \lambda .
$$

Then, the action becomes

$$
S=\int d^{4} x \sqrt{-g}\left[R+\frac{1}{2} \partial_{\mu} \phi \partial^{\mu} \phi-V(\phi)\right],
$$

describing an Einstein-phantom system with an invariant potential. The corresponding Einstein and Klein-Gordon equations are

$$
\begin{gathered}
G_{\mu \nu}=-\partial_{\mu} \phi \partial_{\nu} \phi+\frac{1}{2} g_{\mu \nu} \partial_{\alpha} \phi \partial^{\alpha} \phi-g_{\mu \nu} V \\
\partial_{\alpha} \partial^{\alpha} \phi+\frac{d V}{d \phi}=0 .
\end{gathered}
$$

Therefore, we construct a dynamical solution representing a black hole immersed in a phantom scalar field fluid, and corresponding to

$$
\begin{aligned}
d s^{2}= & a(\eta)^{2}\left[-f(r)^{2} d \eta^{2}+\frac{1}{f(r)^{2}} d r^{2}+S(r)^{2} d \Omega^{2}\right] \\
\phi= & \frac{1}{\lambda} \ln \frac{V_{0}\left(\lambda^{2}+2\right)^{2}}{2\left(\lambda^{2}+6\right)}-\lambda \ln a \\
& -\frac{1}{\sqrt{\lambda^{2}-2}} \ln \left(1-\frac{2 w}{r}\right)
\end{aligned}
$$

where

$$
\begin{aligned}
f & =\left(1-\frac{2 w}{r}\right)^{\frac{\alpha}{2}}, \quad \alpha=-\frac{\lambda}{\sqrt{\lambda^{2}-2}}, \\
S^{2} & =r^{2}\left(1-\frac{2 w}{r}\right)^{1-\alpha}, \quad a=\frac{1}{\eta^{\frac{2}{\lambda^{2}+2}}} .
\end{aligned}
$$

We assume that $\lambda>\sqrt{2}$. Next, let us investigate the physical meaning of the constant $w$. When $\lambda>>\sqrt{2}$, we have $a=1$ and $\alpha=-1$, hence the metric becomes

$$
\begin{aligned}
d s^{2}= & -\left(1-\frac{2 w}{r}\right)^{-1} d \eta^{2}+\left(1-\frac{2 w}{r}\right) d r^{2} \\
& +r^{2}\left(1-\frac{2 w}{r}\right)^{2} d \Omega^{2}
\end{aligned}
$$

The coordinate transformation

$$
y=r\left(1-\frac{2 w}{r}\right)
$$

transforms Eq.(153) into

$$
\begin{aligned}
d s^{2}= & -\left(1+\frac{2 w}{y}\right) d \eta^{2}+\left(1+\frac{2 w}{y}\right)^{-1} d y^{2} \\
& +y^{2} d \Omega^{2}
\end{aligned}
$$

which is the Schwarzschild solution with the mass $M=$ $-w$. Therefore, the parameter $w$ corresponds to the negative mass. Hereafter, we use the symbol $-M$ instead of $w$. 
In order to study whether the singularity can ever be naked during the evolution of the phantom field, we calculate the black hole AH. Using the parameters $M$ and $\alpha$, the phantom black hole solution can be written as

$$
\begin{aligned}
d s^{2}= & \frac{1}{\eta^{\frac{2 \alpha^{2}-2}{2 \alpha^{2}-1}}}\left[-\left(1+\frac{2 M}{r}\right)^{\alpha} d \eta^{2}\right. \\
& +\left(1+\frac{2 M}{r}\right)^{-\alpha} d r^{2} \\
& \left.+r^{2}\left(1+\frac{2 M}{r}\right)^{1+\alpha} d \Omega^{2}\right] .
\end{aligned}
$$

By replacing the conformal time $\eta$ with the comoving time $t$, we can rewrite the metric as

$$
\begin{aligned}
d s^{2}= & -\left(1+\frac{2 M}{r}\right)^{\alpha} d t^{2}+a(t)^{2}\left[\left(1+\frac{2 M}{r}\right)^{-\alpha} d r^{2}\right. \\
& \left.+r^{2}\left(1+\frac{2 M}{r}\right)^{1+\alpha} d \Omega^{2}\right] \\
a(t)= & \left(t_{0}-t\right)^{-\frac{2 \alpha^{2}-2}{\alpha^{2}}} .
\end{aligned}
$$

The scale factor tells us that the constant $t_{0}$ has the physical meaning of the moment at which the Big Rip happens. We have $\alpha<-1$ because of $\lambda>\sqrt{2}$. The exponent $\alpha$ is determined by the slope of the potential. It is apparent that, when $M=0$, the metric (57) describes a phantom-dominated Universe. For simplicity, we set $\alpha=-3$, i.e., $\lambda=3 / 2$. Then, the metric (57) reduces to

$$
\begin{aligned}
d s^{2}= & -\left(1+\frac{2 M}{r}\right)^{-3} d t^{2}+a(t)^{2}\left[\left(1+\frac{2 M}{r}\right)^{3} d r^{2}\right. \\
& \left.+r^{2}\left(1+\frac{2 M}{r}\right)^{-2} d \Omega^{2}\right] \\
a(t)= & \left(t_{0}-t\right)^{-16 / 9} .
\end{aligned}
$$

Using the physical radius $R=\operatorname{ar}(1+2 M / r)^{-1}$ instead of thecoordinate radius $r$, we obtain

$$
\begin{aligned}
& 1+\frac{8 M a}{R}\left(1+\sqrt{1+\frac{8 M a}{R}}\right)^{-1} \\
& -\frac{H R}{32}\left(1+\sqrt{1+\frac{8 M a}{R}}\right)^{5}=0,
\end{aligned}
$$

as the equation of the AH. Here, $H \equiv \frac{d \ln a}{d t}$ is the Hubble parameter. By setting $x \equiv 1+\sqrt{1+\frac{8 M a}{R}}$, one obtains

$$
a M H x^{4}-4 x^{2}+12 x-8=0 .
$$

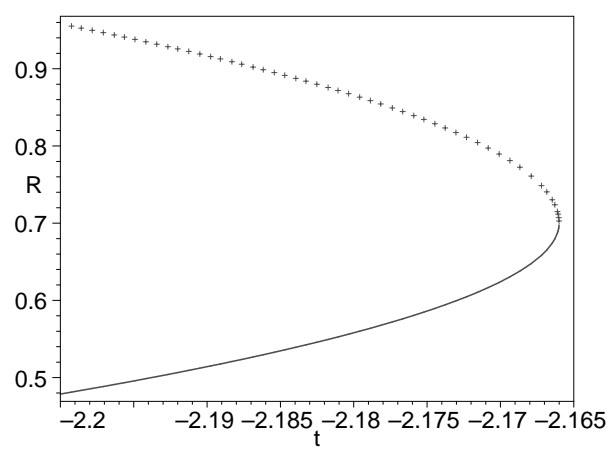

FIG. 2: The black hole apparent horizon (solid line) increases, while the cosmic apparent horizon (dotted line) decreases with the divergence of the phantom field. There is a moment at which the two horizons coincide and after which they disappear, the black hole singularity becoming naked. These plots correspond to $\alpha=-3, t_{0}=1, M=1$.

We find that Eq. (60) has only two physical roots which denote the cosmic $\mathrm{AH} R_{c}$ and the black hole $\mathrm{AH} R_{b}$, respectively. Using Eq. (60) and the definition of $x$, we have plotted the relation between the comoving time $t$ and the AHs in Fig. (2). The latter shows that the black hole apparent horizon increases while the cosmic apparent horizon decreases with the blow-up of the phantom scalar field. There is a moment at which the two horizons coincide and after which both horizons disappear and the black hole singularity becomes naked.

\section{DISCUSSION AND CONCLUSIONS}

The well-known McVittie solution can not describe a black hole embedded in a FRW universe. On the contrary, the new solutions of [17] do: the surface $r=M_{0} / 2$ is not an event horizon but a 2 -sphere contained inside the black hole $\mathrm{AH}$ and a conformal Killing horizon when $M(t)=M_{0} a(t)$. The energy density and pressure are regular at the $\mathrm{AH}$, contrary to the case of the McVittie metric in which the pressure diverges on the would-be horizon. Therefore, we adopt the solution (17) of Faraoni and Jacques [17] to investigate the problem of phantom matter accretion by black holes. We take into account the backreaction, which was ignored in the previous work of Babichev et al. [8]. In a low density background this effect can be safely neglected; however, if the energy density of the background is large (e.g., if it becomes comparable to the black hole density $1 / M_{0}^{2}$ ), the metric describing this black hole will be modified significantly. Therefore, backreaction must be part of the picture.

Using this solution we find that, according to both the quasi-local and the generalized Misner-Sharp mass notions, the black hole mass increases during the cosmic evolution with accretion of the phantom fluid. First of all, one notes that more than one notion of mass can be 
employed, and it is not trivial to identify the correct one (the Hawking-Hayward quasi-local mass has proved to be the most convenient in previous studies [15, 17]); this issue is absent when using a constant mass Schwarzschild black hole as in [8]. The discrepancy between our result of increasing Hawking mass and size of the AH, and the decreasing mass property found in [8] might be explained by the fact that the quasi-local mass does not receive contributions from the negative pressure.

In Faraoni and Jacques [17], there is also a solution (eq. (98)) which yields the opposite result, i.e., the black hole mass decreases with the accretion of phantom fluid. However, that solution requires an outgoing radial energy flux $q=-(P+\rho) u / 2$, which is not easy to interpret for an accreting black hole, and could easily explain the decreasing mass. Here, we have shown that, for an inward energy flux, the black hole mass increases even when phantom energy fluid is accreted.

Unfortunately, it is not easy to compare our solution to the Babichev et al. solution [8] directly, as they have ignored the backreaction effect of expansion, while we have simplified our model of fluid flow instead. The comparison is not straightforward: it is not a simple matter of taking a limit in the relevant equations, because here the relevant black hole mass is taken as the Hawking-Hayward quasi-local mass for the cosmological black hole immersed in a fluid that sources the surrounding FRW universe-this is forced upon us when looking for a meaningful mass concept to use. When comparing with [8], one must replace this quasi-local mass with the Schwarzschild mass and neglect its time dependence, which implies setting $a$ to a constant and, in turn, means that the background fluid becomes a test fluid (it no longer causes the cosmic expansion). The crucial difference between our solutions and that of [8] is that the black hole mass is no longer obtained as a "mass contrast" with respect to the background, but it will include both energy density $\rho$ and pressure $P$ inside the horizon, thus causing a flow of accreted total energy that is negative (however, in this regime, the effect on the Schwarzschild mass is neglected). While we do not doubt that the analysis of [8] is correct in the test-fluid regime, it is its extrapolation to a gravitating fluid and to an expanding universe that we question, and we can only propose some special classes of exact solutions to further the analysis. Because these may be very special, we do not claim that our is the last word on this issue - it is an intermediate step (but a valuable one because exact solutions are rare), and we auspicate that more exact solutions incorporating an explicit radial mass flow be found to discuss this issue further.

The central, and most interesting point of our study, however, is not the behavior of the black hole mass but rather the process in which the black hole $\mathrm{AH}$ expands while the cosmic AH shrinks. When the comoving time derivative of the scale factor approaches the critical value $1 /\left(8 \pi M_{0}\right)$ at the time $t_{*}$ given by Eq. (27), the two horizons coincide. At times $t>t_{*}$ both horizons disappear and the black hole singularity becomes a naked singularity; this happens before the universe reaches the Big Rip. The shrinking of the cosmic apparent horizon has serious consequences. The Bekenstein-Hawking formula $S=A / 4$ [37, 38] tells us that the horizon entropy $S$ is proportional to the area $A$ of its cosmic apparent horizon. So, the entropy of a phantom energy-dominated universe decreases, in violation of the second law of thermodynamics. If the black hole singularity becomes naked in a finite time well before the Big Rip, the Cosmic Censorship Conjecture is violated. By combining these considerations, one could go as far as reaching the conclusion that nature may not permit the existence of phantom energy. There is, however, another consideration to keep in mind: the solution (17) (as well as the similar solutions of [17, 25]) is meant to describe a black hole that has existed forever, not one that is created by gravitational collapse. According to the expression (25) of the $\mathrm{AH}$ radius on which we base our considerations, if this black hole is created together with the universe in a Big Bang singularity, early on the derivative $\dot{a}$ was arbitrarily large and the central singularity was not covered by a horizon, either. Hence, the metric (17) describes a naked singularity (violating the Cosmic Censorship Conjecture) which gets dressed by a horizon shortly after the Big Bang, and evolves as a black hole spacetime until it becomes again a naked singularity (violating Cosmic Censorship again). This bizarre behavior leads one to wonder whether this is a physically meaningful solution. After all, it is well known that naked singularities form during simulations of gravitational collapse, but they are not generic. "Typical" choices of initial data result in the collapse to a black hole and naked singularities appear to be the exception rather than the rule. It could well be that the solution (17) describes a non-physical, fine-tuned situation with bizarre phenomenology. The more general solutions considered in Sec. IV exhibit the same phenomenology and this fact tends to exclude finetuning, but no truly definitive statement can be made at present to this regard due to the paucity of exact solutions describing the physical situation at hand.

Using Eq. (14) and Eq. (23) with $w>-1$, one finds that the black hole $\mathrm{AH}$ first shrinks and then expands, while the cosmic AH always expands with the evolution of the universe, with the cosmic $\mathrm{AH}$ expanding faster than the black hole AH. There was a moment at which the two horizons coincided and before which the singularity was naked. As an example, we have plotted in Fig. 3 the evolution of the cosmic and black hole AHs in a dustdominated universe.

The reason for the black hole $\mathrm{AH}$ shrinking initially is that the density, and thus the expansion speed of the universe, were very large in the vicinity of this coincidence. In this sense, the expansion of the universe "wins" over the attraction of the black hole near this coincidence.

Finally, we comment on the global structure of the cosmological black holes discussed. In Figures 4 and 5 we plot the Penrose-Carter diagrams for both the phantom- 


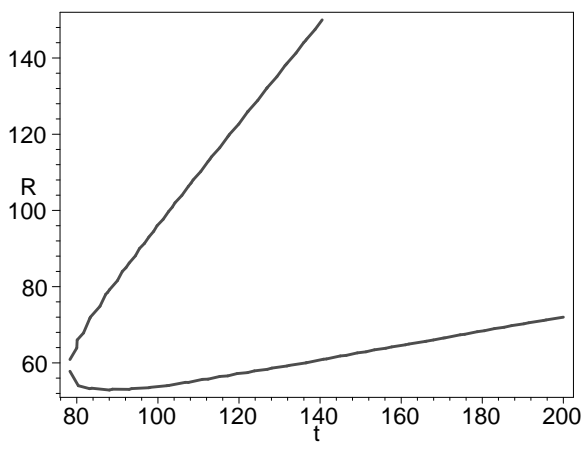

FIG. 3: The black hole apparent horizon (lower line) first decreases and then increases, while the cosmic apparent horizon (upper line) always increases in the dust-dominated Universe. There was a moment at which the two horizons coincided and before which the singularity was naked. The plots correspond to $M_{0}=0.8, a_{0}=1$.

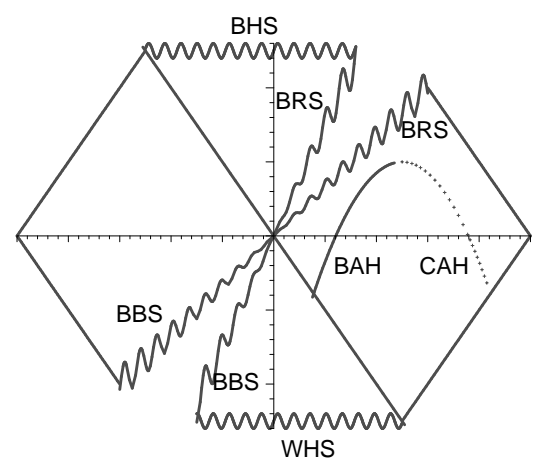

FIG. 4: The Penrose-Carter diagram for a phantomdominated universe with a black hole. The diagram is similar to the one for the Schwarzschild solution except for two sections removed and two cosmic singularities added. For brevity, we only label the black hole singularity (BHS), white hole singularity (WHS), Big Bang singularity (BBS), and Big Rip singularity (BRS) in the diagram. The black hole apparent horizon (BAH) and cosmic apparent horizon (CAH) are also shown.

dominated and the dust-dominated universe with a black hole, respectively. The global structure and Penrose diagram of the Sultana-Dyer solution has been studied in [39], and these are similar for our conformally Schwarzschild solutions in a dust-dominated universe (see Fig. 3 of [39]).

Hence, the singularity will never become naked in a universe dominated by ordinary (or dark) matter. In a universe driven by exotic phantom matter, the black hole $\mathrm{AH}$ also expands. One then wonders which mechanism can make the black hole AH to shrink. Recall that the

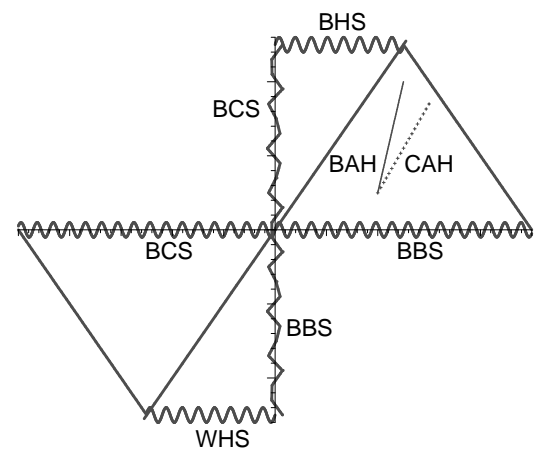

FIG. 5: The Penrose diagram for a dust-dominated universe with a black hole. This diagram also coincides with the one for the Schwarzschild solution, except for two sections removed and two cosmic singularities added. We only label the black hole singularity (BHS), white hole singularity (WHS), Big Bang singularity (BBS), and Big Crunch singularity (BCS) in the diagram. The black hole apparent horizon $(\mathrm{BAH})$ and cosmic apparent horizon $(\mathrm{CAH})$ are also shown.

Schwarzschild anti-de Sitter metric is

$$
\begin{aligned}
d s^{2}= & -\left(1-\frac{2 M_{0}}{r}+H^{2} r^{2}\right) d t^{2} \\
& +\left(1-\frac{2 M_{0}}{r}+H^{2} r^{2}\right)^{-1} d r^{2}+r^{2} d \Omega^{2} .
\end{aligned}
$$

In this case, $H$ is a constant and the black hole $\mathrm{AH}$ is smaller than that of the corresponding asymptotically flat black hole. Accordingly, the generalized MisnerSharp mass is smaller than the one of the hole without cosmological constant. We note that the Schwarzschildanti-de Sitter spacetime has a remarkable difference in comparison with Schwarzschild-de Sitter: its energy density $\rho=-\frac{3}{8 \pi} H^{2}$ is negative. This solution suggests that if a black hole is surrounded by negative and decreasing energy density, its mass will decrease monotonically and will tend to zero in a finite or an infinite cosmic time. However, even though in the solutions considered here the phantom kinetic energy density is negative, its total energy density is positive, hence this would not happen.

\section{Acknowledgments}

We thank a referee for insightful comments leading to improvements in the original manuscript. Thanks go to R. G. Cai, X. Zhang and H. S. Zhang for helpful discussions. This work is supported by the National Science Foundation of China under the Distinguished Young Scholar Grant 10525314, the Key Project Grant 10533010, and Grants No. 10575004, 10573027, and 10663001; by the Shanghai Natural Science Foundation under Grant No. 05ZR14138; by the Chinese Academy of Sciences under grant KJCX3-SYW-N2; and by the Ministry of Science and Technology under the National Ba- 
sic Sciences Program (973) under grant 2007CB815401. V.F. acknowledges the Natural Sciences and Engineering
Research Council of Canada (NSERC).
[1] P.S. Corasaniti, M. Kunz, D. Parkinson, E.J. Copeland and B.A. Bassett, Phys. Rev. D 70, 083006 (2004).

[2] U. Alam, V. Sahni, T.D. Saini and A.A. Starobinsky, Mon. Not. Roy. Astron. Soc. 354, 275 (2004).

[3] R.R. Caldwell, Phys. Lett. B 545, 23 (2002).

[4] G.W. Gibbons, arXiv:hep-th/0302199

[5] S. Nojiri and S.D. Odintsov, Phys. Lett. B 562, 147 (2003).

[6] R.R. Caldwell, M. Kamionkowski, and N.N. Weinberg, Phys. Rev. Lett. 91, 071301 (2003).

[7] D. F. Mota, C. van de Bruck, Astron. Astrophys. 421, 71 (2004); S. Nesseris and L. Perivolaropoulos, Phys. Rev. D 70, 123529 (2004).

[8] E. Babichev, V. Dokuchaev and Yu. Eroshenko, Phys. Rev. Lett. 93, 021102 (2004).

[9] R. Penrose, Riv. Nuovo Cim. 1, 252 (1969) [Gen. Rel. Grav. 34, 1141 (2002)].

[10] S.A. Hayward, S. Mukohyama, and M.C. Ashworth, Phys. Lett. A 256, 347 (1999); S.A. Hayward, Class. Quantum Grav. 15, 3147 (1998).

[11] D. Bak and S.J. Rey, Class. Quantum Grav. 17, L83 (2000).

[12] R.G. Cai and S.P. Kim, J. High Energy Phys. 02, 050 (2005).

[13] X. Zhang, arXiv: 0708.1408; D. Brill, G. Horowitz, D. Kastor, J. Traschen, Phys. Rev. D 49, 840 (1994); H. Saida, T. Harada, and H. Maeda, Class. Quantum Grav. 24, 4711 (2007); M. Nozawa and H. Maeda, arXiv:0710.2709 A. Ashtekar and G.J. Galloway, arXiv:grqc/0503109; A. Ashtekar and B. Krishnan, Living Rev. Rel. 7, 10 (2004); Phys. Rev. D 68, 104030 (2003); Phys. Rev. Lett. 89, 261101 (2006); A. Ashtekar and A. Corichi, Class. Quant. Grav. 17 (2000) 1317 (2000); A. Ashtekar, A. Corichi, and D. Sudarsky, Class. Quant. Grav. 20, 3413 (2003); A. Ashtekar, J. Engle, T. Pawlowski and C. Van Den Broeck, Class. Quant. Grav. 21, 2549 (2004); A. Ashtekar, arXiv:gr-qc/0306115. A. Ashtekar, C. Beetle, and J. Lewandowski, Class. Quant. Grav. 19, 1195 (2002); Phys. Rev. D 64. 044016 (2001); A. Ashtekar, C. Beetle, O. Dreyer, S. Fairhurst, B. Krishnan, J. Lewandowski, and J. Wisniewski, Phys. Rev. Lett. 85, 3564 (2000); A. Ashtekar, S. Fairhurst and B. Krishnan, Phys. Rev. D 62, 104025 (2000); A. Ashtekar, C. Beetle and S. Fairhurst, Class. Quant. Grav. 17, 253 (2000); 16, L1 (1999); A.B. Nielsen and M. Visser, Class. Quantum Grav. 23, 4637 (2006); D. Kotawala, S. Sarkar, and T. Padmanabhan, Phys. Lett. B 652, 338 (2007); M. Nadalini, L. Vanzo, and S. Zerbini, arXiv:07010.2474; M.L. McClure, arXiv:0709.3288 J.A. de Freitas Pacheco and J.E. Horvath, arXiv:0709.1240. D.C. Guariento, J.E. Horvath, P.S. Custodio, and J.A. de Freitas Pacheco, arXiv:0711.3641 P.S. Custodio and J.E. Horvath, Int. J. Mod. Phys. D 14, 257 (2005); I. Booth, Can. J. Phys. 83, 1073 (2005); Z-H, Li and A. Wang, Modern. Phys. Lett. A, 22, 1663 (2007); G. Izquierdo and D. Pavon, Phys. Lett. B 639, 1 (2006); S. Chen and J. Jing, Class. Quantum Grav. 22, 4651
(2005); T. Clifton, D.F. Mota, and J.D. Barrow, Mon. Not. R. Astron. Soc. 358, 601 (2005); ; N. Sakai and J.D. Barrow, Class. Quantum Grav. 18, 4717 (2001); S.A. Hayward, Phys. Rev. D 70, 104027 (2004); Phys. Rev. Lett. 93, 251101 (2004); arXiv:gr-qc/0008071; Phys. Rev. Lett. 81, 4557 (1998); Phys. Rev. D 53, 1938 (1996); Class. Quant. Grav. 11, 3025 (1994); Phys. Rev. D 49, 6467 (1994); arXiv:gr-qc/9303006; S. Mukohyama and S.A. Hayward, Class. Quant. Grav. 17, 2153 (2000); H. Maeda, arXiv:0704.2731; G. Kang, Phys. Rev. D 54, 7483 (1996); T. Jacobson and G. Kang, Class. Quantum Grav. 10, L201 (1993); C.C. Dyer and E. Honig, J. Math. Phys. (NY) 20, 409 (1979); S. Nojiri and S.D. Odintsov, Phys. Rev. D. 70, 103522 (2004).

[14] R. Di Criscienzo, M. Nadalini, L. Vanzo, S. Zerbini, and G. Zoccatelli, Phys. Lett. B 657, 107 (2007).

[15] V. Faraoni, Phys. Rev. D. 76, 104042 (2007).

[16] P. Gonzalez-Diaz, Phys. Rev. Lett. 93, 071301 (2004); S.V. Sushkov and S.-W. Kim, Gen. Rel. Gravit. 36, 1671 (2004); V. Faraoni and W. Israel, Phys. Rev. D 71, 064017 (2005); V. Faraoni, Phys. Lett. B 647, 309 (2007); and references therein.

[17] V. Faraoni and A. Jacques, Phys. Rev. D 76, 063510 (2007).

[18] G.C. McVittie, Mon. Not. R. Astron. Soc. 93, 325 (1933).

[19] P.D. Noerdlinger and V. Petrosian, Astrophys. J. 168, 1 (1971); D.J. Shaw and J.D. Barrow, Phys. Rev. D 73, 123505 (2006); C.J. Gao and S.N. Zhang, Phys. Lett. B 595, 28 (2004); C.J. Gao, Class. Quantum Grav. 21, 4805 (2004); B. Bolen, L. Bombelli and R. Puzio, Class. Quantum Grav. 18, 1173 (2001).

[20] R. Sussman, Gen. Rel. Grav. 17, 251 (1985); M. Ferraris, M. Francaviglia, and A. Spallicci, Nuovo Cimento 111B, 1031 (1996); B.C. Nolan, Class. Quantum Grav. 16, 1227 (1999).

[21] B.C. Nolan, Class. Quantum Grav. 16, 3183 (1999).

[22] B.C. Nolan, Phys. Rev. D 58, 064006 (1998).

[23] M.L. McClure and C.C. Dyer, Class. Quantum Grav. 23, 1971 (2006); Gen. Rel. Gravit. 38, 1347 (2006).

[24] This kind of solution was also hypothesized, but not pursued, in Ref. [14].

[25] J. Sultana and C.C. Dyer, Gen. Rel. Grav. 37, 1349 (2005).

[26] Y. Gong and A. Wang, Phys. Rev. Lett. 99, 211301 (2007).

[27] M. Akbar and R.G. Cai, Phys. Rev. D 75, 084003 (2007).

[28] R.G. Cai and L.M. Cao, Phys. Rev. D 75, 064008 (2007).

[29] R.G. Cai and L.M. Cao, Nucl. Phys. B 785, 135 (2007).

[30] M. Akbar and R.G. Cai, Phys. Lett. B 648, 243 (2007).

[31] A. Sheykhi, B. Wang and R.G. Cai, Phys. Rev. D 76, 023515 (2007).

[32] A. Sheykhi, B. Wang and R.G. Cai, Nucl. Phys. B 779, 1 (2007).

[33] S.A. Hayward, Phys. Rev. D 49, 831 (1994).

[34] C.M. Misner and D.H. Sharp, Phys. Rev 136, B571 (1964).

[35] O.A. Fonarev, Class. Quantum Grav. 12, 1739 (1995). 
[36] Y. Kitada and K. Maeda, Phys. Rev. D 45, 1416 (1992); Y. Kitada and K. Maeda, Class. Quantum Grav. 10, 703 (1993); A.R. Liddle, A. Mazumdar and F.E. Schunck, Phys. Rev. D 58, 061301 (1998); K.A. Malik and D. Wands, Phys. Rev. D 59, 123501 (1999); A.A. Coley and R.J. van den Hoogen, Phys. Rev. D 62, 023517 (2000); Z-K. Guo, Y-S. Piao, R-G. Cai and Y-Z. Zhang, Phys.
Lett. B 576, 12 (2003).

[37] J. Bekenstein, Phys. Rev. D 12, 3077 (1975).

[38] S.W. Hawking, Phys. Rev. D 13, 191 (1976).

[39] H. Saida, T. Harada, and H. Maeda, Class. Quantum Grav. 24, 4711 (2007). 